\title{
Experimental Characterization of Intermolecular Multiple-Quantum Coherence Pumping Efficiency in Solution NMR
}

\author{
Huiming Zhang, ${ }^{*} \dagger$ Natalia Lizitsa, $\ddagger$ Robert G. Bryant,* and Warren S. Warren $\ddagger$ \\ *Department of Chemistry, University of Virginia, Charlottesville, Virginia 22901; $\$$ Department of Chemistry, Princeton University, Princeton, \\ New Jersey 08544; and $\dagger$ Center for MR Research, ENH Research Institute, Northwestern University, Evanston, Illinois 60201
}

Received August 14, 2000

\begin{abstract}
The behavior of intermolecular multiple-quantum coherences in a variety of simple liquids with different chemical and magnetic properties is investigated experimentally and modeled by numerical simulations based on modified B loch equations. The effects of spin concentration, temperature, intramolecular conformational flexibility, chemical exchange, and spin-spin coupling on the formation of high-order coherences are examined. It is shown that any process that makes the Larmor frequency time-dependent may interfere with the formation of these coherences. G ood agreement is achieved between experiments and simulation, using independently known values of the magnetization density, the rate constants for translational diffusion, spin-spin and spin-lattice relaxation, and radiation damping. $\odot 2001$ Academic Press

Key Words: multiple quantum; CR AZE D; intermolecular dipolar fields; echoes.
\end{abstract}

\section{INTRODUCTION}

Observations of multiple-quantum (MQ) coherences in both liquid- and solid-state magnetic resonance contribute significantly to the analytical power of the spectroscopy by providing highly selective spectral editing filters and amplifying effects of frequency differences in chemical exchange and translational diffusion experiments (1-7). The simplest conventional experimental approaches for MQ excitation and detection involve three RF pulses $\left(90_{x}-\tau-90_{x}-t_{1}-90_{x}-t_{2}\right)$. If $\tau$ is chosen to be at least comparable to the reciprocal of a typical coupling (50-500 $\mathrm{ms}$ in isotropic liquids, $1-10 \mathrm{~ms}$ in oriented materials), this sequence gives resonances corresponding to "forbidden" $(\Delta M \neq 1)$ transitions in the indirectly detected dimension. Even seemingly highly forbidden transitions (such as the six-spin flip in oriented benzene) can be seen in MQ experiments with transition intensities comparable to the allowed transitions. These experiments depend on intramolecular couplings and this intramolecular isolation has been very powerful in the design of experiments that provide key structural or dynamic information.

It is now possible to observe intermolecular multiple-quantum effects in simple liquids using pulse sequences that involve as few as two RF pulses, as pointed out by Warren and collaborators $(8-11)$ some time ago. These multiple-quantum coherences may be observed even when the spin system of the observed molecule is simple such as that in water, for which intramolecular multiple-quantum effects are not generally observed. Thus, it is now possible to take advantage of multiplequantum spectroscopy techniques even for molecules with very simple spin systems that provide only a single resonance line. The simplest basic pulse sequence, called a CRAZED sequence, is shown in Fig. 1a. It consists of a $90^{\circ}$ pulse followed by a magnetic field gradient pulse and an evolution period, $t_{1}$. Then a second $90^{\circ}$ pulse is applied followed by a second gradient pulse of $n$ times the duration of the first. The resulting signal is detected during the second evolution period, $t_{2}$. Peaks in the indirectly detected dimension have the experimental properties of multiple-quantum coherences between molecules that are significantly separated in space. Thus, these coherences depend on fundamentally different couplings from those commonly exploited and provide new opportunities for analytical applications that include spectral editing and characterization of inter- and intramolecular chemical dynamics.

The application of the magnetic field gradients in the pulse sequence provides a spatial encoding of the resonant spins in the sample that breaks the angular averaging of the dipolar fields. The coherences between spatially separated molecules are made observable by the resultant dipolar fields associated with the precessing magnetization. Motions of the spins during the pulse sequence may destroy the coherence and reduce the signal intensity in a way similar to multiple-quantum experiments that develop using three RF pulses. Because the coherence orders potentially developed by the long-range intermolecular coupling of dipolar fields may be very high, the sensitivity to diffusive motions may be significantly enhanced which will permit study of slow translational motions using modest magnetic field gradients that are too slow to be studied using conventional methods. As Johnson initially pointed out, applications of diffusion weighted spectroscopy may be very useful as a spectral editing tool that couples structural analysis with effective local transport dynamics (12). What is potentially significant in the CRAZED-type experiment is that the coherence order that may be created does not depend on the 
$\mathbf{a}$

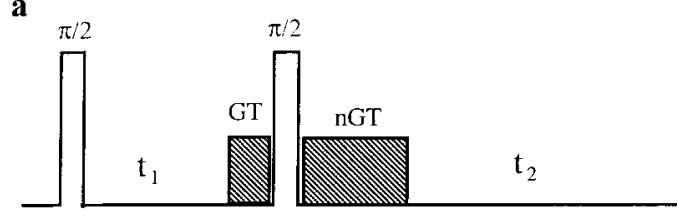

$\mathbf{b}$

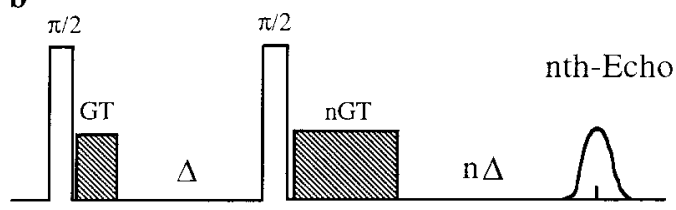

c

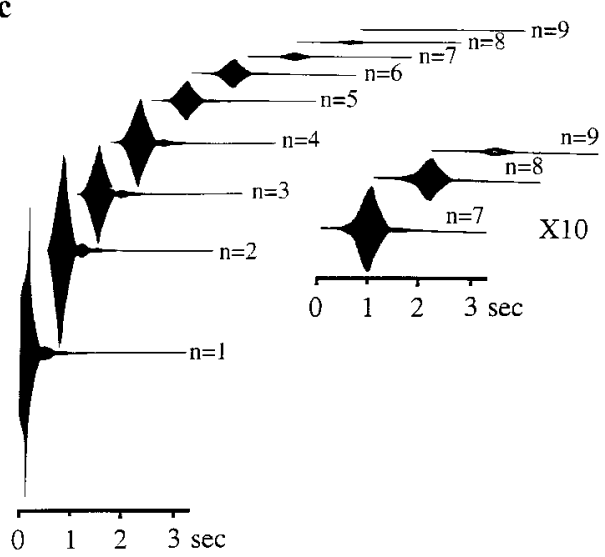

FIG. 1. (a) The CRAZED pulse sequence. This is a two-pulse COSY sequence modified with an $n$-quantum filter. The first $90^{\circ}$ pulse and gradient create MQ coherence, while the second makes it observable. The second gradient selects the coherence order. (b) Modified version of the CRAZED sequence used in this work. The evolution periods $t_{1}$ and $t_{2}$ were replaced with fixed delays, $\Delta$ and $n \Delta$, respectively. The delay, $\Delta$, was set to $150 \mathrm{~ms}$ except for Sephadex G-10 experiments where a 10-ms delay was used. The strength of the gradient, $G$, was set to $3.27 \mathrm{G} / \mathrm{cm}$; the duration of the gradient was $1 \mathrm{~ms}$ (see text). (c) MQ coherences obtained with the pulse sequence represented in water.

number of spins in the molecule. As we show experimentally below, even for water, coherences of order 10 may be detected and exploited.

The use of magnetic field gradients is now common in many NMR pulse sequences. As a consequence, multiple-quantum coherences arising from dipolar field effects may inadvertently contribute to spectra and produce artifacts that may not only be confusing but also lead to incorrect interpretations. However, the interest in these phenomena derives from a more fundamental possibility of exploiting the new information associated with the long-distance effects associated with the collective dipolar field. Because the coherences are developed between spins separated in space by distances that are large compared with molecular dimensions, there are new opportunities for exploring effects between spins that are in different regions of an inhomogeneous sample. Such situations are common in biological systems but may include any system in which there is a concentration gradient. Cross-peaks between spins that are in compartments that are separated by a glass boundary have already been reported, as has contrast enhancement for imaging in vivo.

The new opportunities provided by these "dipolar field effects" are not without limit, and the purpose of the present work is to examine the basic features of the effects in simple chemical systems and provide a reasonably quantitative description of the effects. In particular, we examine the effects of chemical complexity, spin concentration, intramolecular and intermolecular dynamics, and temperature on the development and detection of multiple-quantum coherences created by the CRAZED-type sequence. An important aspect was to see whether the currently used models can provide a quantitative description of the experimental observations, which are seemingly counterintuitive and were the subject of some discussion in the literature (13-15). Since pulse sequences containing the same blocks as CRAZED are routinely used in multidimensional NMR spectroscopy, understanding these coherences is important if interpretive errors are to be avoided. Further, these effects may have interesting applications in their own right in imaging and removal of inhomogeneous broadening $(16,17)$. We show that any process that makes the Larmor frequency time-dependent may interfere with the formation of these echoes. The echo amplitude responds to chemical exchange events in a way that is qualitatively similar to responses to pulse sequences employing very different phase refocusing schemes but appears to be more sensitive for the high-order coherences.

Reference (10) noted that the coherences created between molecules at significant separations behaved like more familiar intramolecular multiple-quantum coherences and used the language of multiple-quantum coherences to interpret the observed data. However, the only quantitative treatment used modified nonlinear Bloch equations that invoked the "dipolar demagnetizing field," observed in a completely different context over a decade earlier $(18-20)$. What Warren and coworkers have come to call the "classical treatment" was replaced in Ref. (11) by a density matrix treatment that explicitly contained intermolecular multiple-quantum operators. In either treatment, the necessary couplings are provided by the dipolar interactions between distant spins in solution. The results prompted a careful exploration of the theoretical framework and the relationship between classical and density matrix treatments for interpreting these affects as genuine multiple-quantum coherences $(21,22)$. It is now clear that, in solution, either method gives essentially the same answer; the density matrix treatment usually gives better intuition while the classical treatment is more convenient for calculations.

For the experiments reported here we used the sequence represented in Fig. 1 b. A $90^{\circ}$ pulse is followed by a $Z$-gradient pulse to encode the induced coherences with a phase increment proportional to the coherence order. The delay, $\Delta$, is set to 150 ms except for the Sephadex G-10 experiments, where a 10-ms delay was used because of the very short signal decay times. A second $90^{\circ}$ pulse is applied to make the coherence observable 
and the coherence of order $n$ is selected with a second $z$-gradient pulse of the same amplitude but $n$ times the duration of the first. Figure 1c shows the echoes detected in water using this pulse sequence, which we call diffusion weighted CRAZED because the substantial value of $\Delta$ emphasizes the effects of translation.

\section{EXPERIMENTAL}

Liquid samples were sealed in a 1.5-mm-id, 0.3-mm-wallthickness capillary tube (Kimble Glass Co.) and were placed in a 5-mm NMR tube filled with $99.9 \% \mathrm{D}_{2} \mathrm{O}$ (Cambridge Isotope Laboratories, Woburn, MA). Deuterated solvents (100\%), $\mathrm{C}_{6} \mathrm{D}_{6}, \mathrm{CD}_{3} \mathrm{CN}$, and $\mathrm{CD}_{3} \mathrm{C}_{6} \mathrm{D}_{5}$, were purchased from Aldrich (Aldrich Chemical Co., Milwaukee, WI). Mixing of liquid samples was done by volume.

2-Chloro-2-methylpropane (99\%), 2,3-butanedione (97\%), 1,2-dichloroethane (99\%), $n$-hexane $(99.9 \%)$, cyclohexene (99\%), anhydrous hydrazine (98\%), cyclopentane (HPLC grade), cyclohexane (99.9\%), and cyclooctane (99\%) were obtained from (Aldrich Chemical Co.). $\mathrm{N}$-Decanol (99\%) and dichloromethane (99.9\%) were purchased from Sigma (Sigma Chemical Co., St. Louis, MO). These liquids were used without further purification.

Sephadex G10, G25, G50, and G100 (Sigma Chemical Co.) were soaked in $\mathrm{pH} 5$ water for $10 \mathrm{~h}$. The hydrated gels were placed in 5-mm NMR tubes which contained $\mathrm{D}_{2} \mathrm{O}$ sealed in a 1.5-mm-id, 0.3-mm-wall-thickness capillary tube to provide a deuterium signal for the field-frequency lock signal.

Gels (7\%) of bovine serum albumin (BSA) (Sigma Chemical Co., A-7030), were cross-linked with glutaraldehyde by mixing $1.6 \mathrm{~mL}$ of $2.0 \mathrm{mM}$ albumin in $10 \mathrm{mM}$ phosphate buffer at pH 7 with $1.3 \mathrm{~mL}$ of $25 \%$ aqueous glutaraldehyde (Sigma Chemical Co.) at ice temperature. The gels or $7 \%$ BSA solution were placed in a 5-mm NMR tube which contained $\mathrm{D}_{2} \mathrm{O}$ sealed in a $1.5-\mathrm{mm}$-id, $0.3-\mathrm{mm}$-wall-thickness capillary tube to provide a deuterium signal for the field-frequency lock.

All experiments were performed with a Varian Unity-Plus NMR spectrometer (Varian Associates, Inc., Palo Alto, CA) operating at a proton resonance frequency of $500 \mathrm{MHz}$ with a Varian triple-resonance probe that provided a magnetic field gradient in the direction of the magnetic field. The proton $90^{\circ}$ pulse width was $9.4 \mu \mathrm{s}$, the calibrated gradient strength was $3.27 \mathrm{G} / \mathrm{cm}$, and the recycle delay was $10 \mathrm{~s}$. The duration of the first gradient pulse was set to $1 \mathrm{~ms}$ and the length of the second gradient pulse was adjusted according to the selected coherence order. The standard delay time, $\Delta$, was $150 \mathrm{~ms}$ in all samples except the Sephadex samples and the serum albumin samples for which it was reduced to 10 and $20 \mathrm{~ms}$, respectively.

\section{THEORETICAL BACKGROUND}

\section{A. General Properties of the Distant-Dipole Field}

The dipolar interaction between two nuclei is proportional to $\left(3 \cos ^{2} \theta-1\right) / r^{3}$, where $\theta$ is the angle between the internuclear vector and the direction of the applied magnetic field, and $r$ is the internuclear separation. The multitude of dipolar couplings in a solid results in a significantly broadened resonance line. However, for nearby spins in a liquid, the rapid rotational and translational diffusive motions change the internuclear vectors rapidly compared with the strength of the couplings. Because $\left\langle\left(3 \cos ^{2} \theta-1\right) / r^{3}\right\rangle=0$, the dipolar couplings are expected to disappear. For pairs of spins that diffuse spatially over distances significantly greater than their initial separation, this argument is correct and accounts for the general observation that dipolar splittings are not observed in most NMR spectra of isotropic liquid phases. However, if the separation between the spins increases, the amount of motion required to effect a complete average of the coupling also increases. For water at room temperature, the diffusion coefficient, $D$, is about $2 \times$ $10^{-5} \mathrm{~cm}^{2} \mathrm{~s}^{-1}$ and spins diffuse approximately $110 \mu \mathrm{m}$ in a second, which is approximately the time needed for acquisition of a typical free induction decay signal. Because sample dimensions are generally much larger than $100 \mu \mathrm{m}$, diffusive motion does not average the dipolar coupling between most spins in the sample. The usual argument that the long-distance dipolar effects are small rests on the fact that the coupling decreases as $1 / r^{3}$. However, the probability that there is a spin at distance $r$ increases in proportion to $r^{2}$, so that the total intermolecular dipolar coupling decreases as only $1 / r$. Thus, neglecting the angular dependence, the integral over the whole sample diverges.

The angular dependence of the dipolar coupling is the reason why these long-range intermolecular effects are not usually observed. For a spherical sample of an isotropic liquid, the spins are distributed symmetrically, and the sum over all additional interactions such as that of the spins with a magnetic field gradient or long-range dipolar fields vanish. In well-filled cylindrical sample tubes, the residual field gradients are small and the effects not often noticeable. If the sample is not spherical or if the magnetization is modulated by an additional interaction, the angular averaging does not eliminate the dipolar effects. The magnetic field gradient pulses in a CRAZED or similar pulse sequence defeat the angular averaging and the dipolar effects may reappear.

In the classical picture, the interaction between spins is handled by introducing an additional field $B_{\mathrm{d}}(\mathbf{r})$, called the "dipolar demagnetizing field" (12-14) or the "distant dipole field" (the latter term will be used here):

$$
\begin{aligned}
\mathbf{B}_{\mathrm{d}}(\mathbf{r})= & \frac{\mu_{0}}{4 ð} \int d^{3} r^{\prime} \frac{1-3 \cos ^{2} \theta_{r r^{\prime}}}{2\left|\mathbf{r}-\mathbf{r}^{\prime}\right|^{3}} \\
& \times\left[3 M_{z}\left(\mathbf{r}^{\prime} \wedge \mathbf{z}-\mathbf{M}\left(\mathbf{r}^{\prime}\right)\right)\right] .
\end{aligned}
$$

Equation [1] gives the secular part of the local magnetic field at position $\mathbf{r}$ generated by the direct dipole-dipole interaction between distant spins. This interaction survives diffusional 
averaging in solution when the distance $\mathbf{r}$ is larger than the distance molecules diffuse on the time scale of the evolution periods in the NMR pulse sequence (1). Equation [1] is then used to modify the normal form of the Bloch equations, giving an additional contribution $\left(\mathbf{M} \times \gamma \mathbf{B}_{\mathrm{d}}(\mathbf{r})\right)$ to $d \mathbf{M} / d t$. If the magnetization $\mathbf{M}(\mathbf{r})$ varies only in a single direction $\mathbf{s}$ (as can happen if gradient pulses are only applied in a single direction), $\mathbf{B}_{\mathrm{d}}(s)$ may be reduced to the much simpler form

$$
\begin{aligned}
\mathbf{B}_{\mathrm{d}}(s) & =\mu_{0} \Delta_{s}\left(M_{\mathrm{z}}(s) \hat{\mathbf{z}}-\frac{1}{3} \mathbf{M}(s)\right) ; \\
\Delta_{s} & =\left[3(\hat{\mathbf{s}} \cdot \hat{\mathbf{z}})^{2}-1\right] / 2,
\end{aligned}
$$

which depends only on the local value of the magnetization. Corrections for residual magnetization have been presented elsewhere (14) and are included in the simulations presented in this work.

\section{B. Expected Behavior of CRAZED Signals}

Our experiments test a wide range of relaxation time values, diffusion rates, and magnetization densities and, in the end, we will compare the experimental results to simulations. However, we can extend previously published analytical results to give a reasonable estimate of the expected behavior. Ignoring relaxation and diffusion, the $n$-quantum signal behaves as a Bessel function of corresponding order,

$$
M_{n}^{+}=i^{n-1} M_{0} n\left(\frac{\tau_{\mathrm{D}}}{t_{2}}\right) J_{n}\left(-\frac{t_{2}}{\tau_{\mathrm{D}}}\right),(n \neq 0),
$$

where $M_{0}$ is the equilibrium Boltzmann magnetization, $n$ is the coherence order, $\tau_{\mathrm{D}}=\left(\gamma \mu_{0} M_{0}\right)^{-1}$ is the dipolar time (which may be thought of as the inverse of the precession frequency in the effective dipolar field), $t_{2}$ is the evolution time (equal to $n \Delta$ for this pulse sequence), and $J_{n}$ is the Bessel function of order $n$. When the argument $x$ of the Bessel function is much less than unity (which corresponds to the limit $t_{2} \ll n \tau_{\mathrm{D}}$ ), the Bessel function may be approximated as

$$
J_{n}=\frac{1}{n !}\left(\frac{x}{2}\right)^{n}
$$

In this case, the intensity of the $n$-quantum coherence, $S_{n}$ is proportional to the $n$th power of the equilibrium Boltzmann magnetization:

$$
\left|M_{n}^{+}\right|=\left(\gamma \mu_{0}\right)^{(n-1)} M_{0}^{n} \frac{1}{2(n-1) !}\left(\frac{t_{2}}{2}\right)^{n-1}
$$

Due to inhomogeneous broadening the signal is lost following
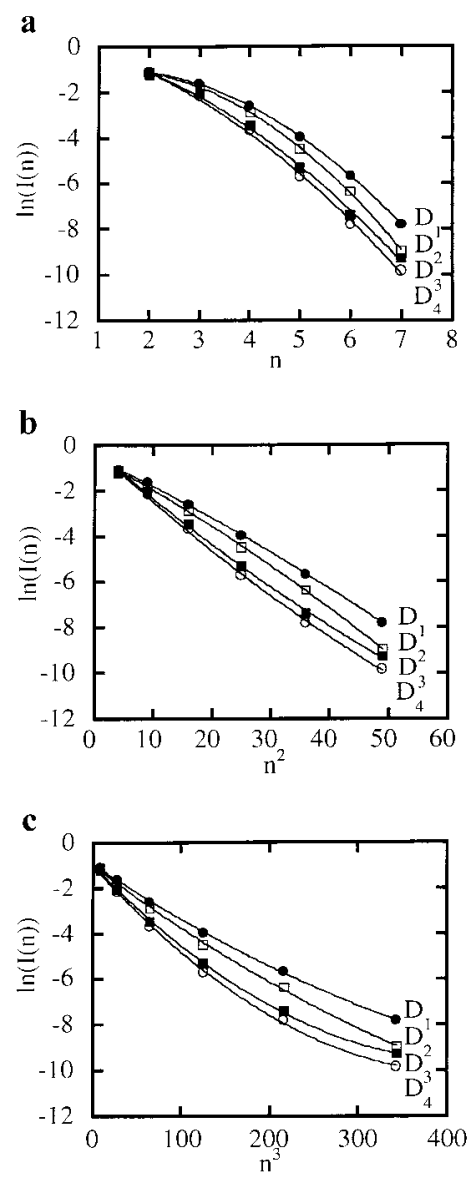

FIG. 2. The logarithm of the simulated MQ signal intensity as a function of coherence order (a), squared coherence order (b), and cubed coherence order (c). Different curves refer to different diffusion coefficients. All simulations were made with the same parameters as experiments except for the strength of the gradient, which was set to $10 \mathrm{G} / \mathrm{cm}$ to make functional behavior more pronounced.

the first pulse but subsequent pulses refocus an echo at $t_{2}=$ $n \Delta$. Still ignoring relaxation and diffusion, the echo intensity is

$$
\frac{\left|M_{n}^{+}\right|}{M_{0}}=\frac{\left(\gamma \mu_{0} M_{0} \Delta\right)^{(n-1)}}{2^{n}} \frac{1}{(n-1) !}(n)^{n-1} .
$$

Equation [6] gives nearly a straight line in a graph of the log of the signal versus $n$. This does not agree with experiment (Fig. 2 ), which more commonly shows a nearly straight line with the $\log$ of the signal versus $n^{2}$.

The dependence on $n^{2}$ is due to relaxation and translational diffusion. The effects of relaxation are most easily visualized in the quantum picture (18). The dominant pathway for generation of observable signal is evolution of intermolecular $n$-quantum coherences (e.g., $I_{x 1} I_{y 2} \ldots I_{y n}$ ) during the interval $\Delta$. This $n$-quantum operator decays by $\exp \left(-n \Delta / T_{2}\right)$ during the interval $\Delta$. Such coherences are partially converted into one-quantum, $n$-spin coherences (e.g., $I_{x 1} I_{z 2} \ldots I_{z n}$ ) by the 
second $90^{\circ}$ pulse and require $(n-1)$ dipolar couplings to become observable magnetization (e.g., $I_{x 1}$ or $I_{y 1}$ ). During $t_{2}=$ $n \Delta$ the one-quantum, $n$-spin coherence decays by $\exp \left(-t_{2} /\right.$ $\left.T_{2}\right) \exp \left(-(n-1) t_{2} / T_{1}\right)=\exp \left(-n \Delta / T_{2}\right) \exp (-(n-1) n \Delta /$ $\left.T_{1}\right)$. The total decay is then $\exp \left(-2 n \Delta / T_{2}\right) \exp (-(n-1) n \Delta /$ $T_{1}$ ), which makes the log of the signal nearly proportional to $n^{2}$ if $T_{1} \approx T_{2}$.

In most of our experiments, however, the dominant signal decay mechanism is diffusion. To understand the effects of diffusion, recall that the dipolar coupling is only capable of creating observable magnetization because the first magnetic field gradient destroys the effective symmetry of the magnetization. For example, in an $n$-quantum CRAZED experiment, the $n$-quantum, $n$-spin operator $I_{x 1} I_{y 2} \ldots I_{y n}$ picks up a phase shift during the first gradient pulse of $\exp \left(i \gamma G T\left(z_{1}+z_{2}+\right.\right.$ $\left.\ldots z_{n}\right)$ ). The second gradient pulse (with $n$ times the duration) gives a phase shift of $\exp \left(n i \gamma G T z_{1}\right)$ to the $n$-spin, one-quantum operators $I_{x 1} I_{z 2} \ldots I_{z n}$. The net effect is a partially refocused term of the form

$$
I_{x 2} I_{z 2} \ldots I_{z n} \cos \left(\gamma G T\left(z_{1}-z_{2}\right)\right) \ldots \cos \left(\gamma G T\left(z_{1}-z_{n}\right)\right)
$$

which depends only on relative position and is made observable by $(n-1)$ dipolar couplings $D_{i j}$.

Diffusion along the direction between spins 1 and $n$ at any time after the first gradient pulse destroys this labeling. Decay of the modulated operators can be calculated by the usual diffusion relations as $\exp \left(-2^{1 / 2} D(\gamma G T)^{2} t\right)$. In the presence of inhomogeneous broadening, signal is observable only about the echo maximum, and the total evolution time for the $n$ quantum CRAZED is approximately $(n+1) \Delta$; the $n$-quantum CRAZED requires $(n-1)$ independent dipolar couplings to convert the $(n-1)$ modulated terms into observable signal. The net effect is a predicted decay of $\exp \left(-2^{1 / 2} D(\gamma G T)^{2}(n-\right.$ $1)(n+1) \Delta)$. In the limit where diffusional decay dominates all other terms (easily achieved in practice for the long evolution times here), the log of the signal is expected to be proportional to $n^{2}$. This is very similar to the dependence of conventional multiple-quantum echoes utilized in the context of translational diffusion experiments (23-27). One practical way to view this result is that observing coherences of order $n$ increases the effective gradient strength by the factor $n$. Therefore, slow diffusive motions may be studied without resort to large gradients, provided other effects such as very short $T_{2}$ values do not quench the coherence. A practical case may be water diffusing in a bounded but tortuous medium.

Numerical simulations were based on the solution of the modified Bloch equations, using independently determined parameters such as relaxation times, diffusion coefficients, and proton densities. The time evolution of the uncoupled spins including relaxation processes is described by the Bloch equations:

$$
\frac{d \mathbf{M}}{d t}=\gamma \mathbf{M} \times \mathbf{B}-\frac{\left(M_{x} \hat{\mathbf{x}}+M_{y} \hat{\mathbf{y}}\right)}{T_{2}}-\frac{\left(M_{z}-M_{0}\right) \hat{\mathbf{z}}}{T_{1}} .
$$

The magnetic field $\mathbf{B}$ in Eq. [7] includes the distant-dipolar field $\mathbf{B}_{\mathrm{d}}(\mathbf{r})$ (Eq. [2]) after a correction for any residual magnetization (16), which for $z$-modulated magnetization gives

$$
\mathbf{B}_{\mathrm{d}}(s)=\mu_{0}\left\{\left(M_{\mathrm{z}}(s)-\left\langle M_{\mathrm{z}}\right\rangle\right) \hat{\mathbf{z}}-\frac{1}{3}(\mathbf{M}(s)-\langle\mathbf{M}\rangle)\right\} .
$$

It also includes an additional field $\mathbf{B}_{\mathrm{r}}$ which is produced by the current induced in the coil by the oscillating magnetization (this is known as radiation damping) (28). It can be expressed as

$$
\mathbf{B}_{\mathrm{r}}=\frac{\left\langle M_{y}\right\rangle}{\gamma M_{0} \tau_{\mathrm{r}}} \hat{\mathbf{x}}+\frac{\left\langle M_{x}\right\rangle}{\gamma M_{0} \tau_{\mathrm{r}}} \hat{\mathbf{y}}, \quad \tau_{\mathrm{r}}=\frac{1}{\gamma \mu_{0} M_{0}(\eta Q / 2)},
$$

where $\tau_{\mathrm{r}}$ is the radiation damping time, $\eta$ is the filling factor, and $Q$ is the quality factor of the probe. In contrast to the distant dipolar field, $\mathbf{B}_{\mathrm{r}}$ is position-independent and depends on the average magnetization only.

The Bloch equations modified to include the dipolar demagnetizing field, radiation damping, diffusion, and relaxation processes become

$$
\begin{aligned}
\frac{d \mathbf{M}(z)}{d t}= & \gamma\left[\mathbf{M}(z) \times \mathbf{B}_{0}(z)\right]+\gamma\left[\mathbf{M}(z) \times \mu_{0} M_{z}(z) \hat{\mathbf{z}}\right] \\
& +\frac{\left[\mathbf{M}(z) \times\left\langle M_{y}\right) \hat{\mathbf{x}}\right]}{M_{0} \tau_{\mathrm{r}}}+\frac{\left[\mathbf{M}(z) \times\left\langle M_{x}\right\rangle \hat{\mathbf{y}}\right]}{M_{0} \tau_{\mathrm{r}}} \\
& -\frac{\left(M_{x} \hat{\mathbf{x}}+M_{y} \hat{\mathbf{y}}\right)}{T_{2}}-\frac{\left(M_{z}-M_{0}\right) \hat{\mathbf{z}}}{T_{1}}+D \frac{d^{2} \mathbf{M}(z)}{d z^{2}}
\end{aligned}
$$

Here $\mathbf{B}_{0}(z)=(\hbar \Delta \omega / \gamma+G z) \hat{\mathbf{z}}$ is the magnetic field due to gradients and resonance offset and $D$ is the translational diffusion coefficient. In our simulations these equations were numerically integrated using the fifth-order Cash-Karp RungeKutta method; the procedure details have been described in previous studies (14). This one-dimensional model assumes uniformly modulated magnetization (except for the observed signal) and thus some discrepancy between simulated and experimental results is expected because of intrinsic magnetic field inhomogeneity. We have recently extended our simulations to include three-dimensional magnetization structure $(29,30)$, which would be feasible here if the exact structure of the residual magnetic field inhomogeneity were known; however, the agreement between the dynamics predicted by Eq. [9] and experiment is reasonable. 


\section{COMPARISON WITH EXPERIMENT}

The results of many experiments using the pulse sequence of Fig. 1 are generally linear when the logarithm of the $n$ th-order signal intensity is plotted against $n^{2}$. This dependence is consistent with numerical simulations carried out for a range of diffusion constants while other experimental parameters are held constant. The logarithm of the signal intensity vs $n$ and $n^{3}$ show significant deviations from linearity. Thus, we summarize data by plotting the logarithm of the echo amplitude vs the square of the coherence order.

\section{A. Magnetization Density Effects}

The signal intensity of the $n$th echo is strongly dependent on the magnitude of the net magnetization and is a function of both the static magnetic field strength and the concentration of the observed spins. Figure 3 a shows the results of diluting the water proton spin concentration with deuterium. The intensity and the number of detectable high-order coherences decreases significantly as the proton spin concentration drops. The dashed lines represent the experimental data while the solid lines were simulated numerically as described above. The simulations describe the data well, except for large values of $n^{2}$ in a few cases. We believe this discrepancy is due to intrinsic static field inhomogeneity, since the simulations used a one-dimensional model that assumes uniformly modulated magnetization (except for the observed signal). The offset frequency of the $n$-quantum signal and the acquisition delay in the CRAZED experiment are $n$ times larger than that of the one-quantum and thus, the signal is $n$ times more sensitive to the effect of inhomogeneity. As expected, the discrepancy between experimental and simulated results in Fig. 3a is larger for the high-order coherences than that of the smaller orders.

Simulations for samples with different deuterium concentrations were made with the same longitudinal and transverse relaxation times as those for pure water, although these parameters increase significantly with increasing of deuterium mole fraction. However, since $T_{1}, T_{2} \gg \tau_{\mathrm{D}}$ even for dilute samples they have no influence on observed spin dynamics and thus the differences in relaxation rates for the samples with different deuterium mole fraction may be neglected.

Equation [3] implies that for two spin concentrations, $M_{01}$ and $M_{02}$, the ratio of $n$ th-order echo intensities is given by Eq. [11],

$$
\ln \frac{S_{n 1}}{S_{n 2}} \propto n \ln \frac{M_{01}}{M_{02}},
$$

where $S_{n 1}$ and $S_{n 2}$ are the intensities of the echoes of coherence order $n$, and $M_{01}$ and $M_{02}$ are the equilibrium magnetizations associated with spin concentrations, 1 and 2 . If this relationship a

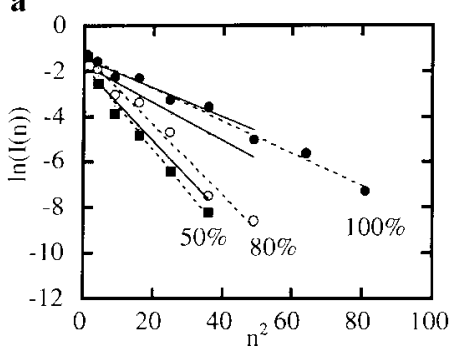

b

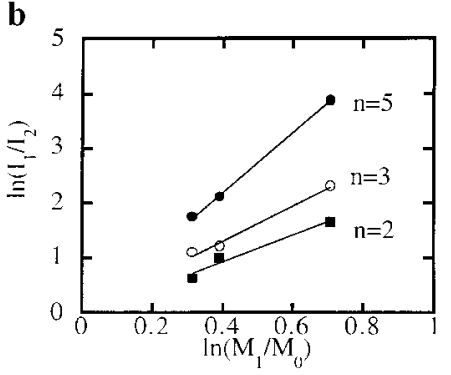

c

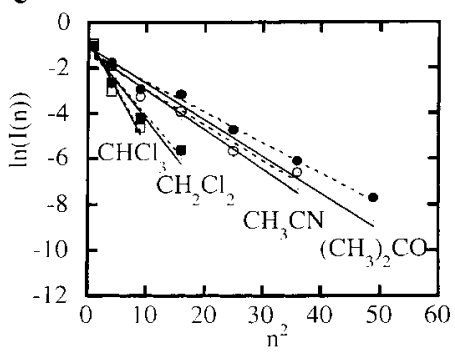

FIG. 3. (a) The logarithm of the MQ coherence intensity vs squared coherence order for water samples with different deuterium mole fractions. The solid lines refer to the results of the simulations, while the dashed lines connect the experimental points. The simulations were made with the following parameters: $T_{1}=2.1 \mathrm{~s}, T_{2}=1.8 \mathrm{~s}, D=1.83 \cdot 10^{-5} \mathrm{~cm}^{2} / \mathrm{s}$. (b) The logarithm of the relative MQ signal intensity as a function of the logarithm of the relative equilibrium magnetization. The slopes of the lines correspond to the coherence order, in accordance with Eq. [11]. (c) The logarithm of the MQ coherence intensity vs squared coherence order for a number of simple solvent systems that present a single resonance line. The dashed lines connect the experimental points; the solid lines refer to the simulations, which use the following parameters: $\mathrm{CHCl}_{3}: T_{1}=1.8 \mathrm{~s}, T_{2}=1.5 \mathrm{~s}, D=1.15 \cdot 10^{-5}$ $\mathrm{cm}^{2} / \mathrm{s} ; \mathrm{CH}_{2} \mathrm{Cl}_{2}: T_{1}=1.5 \mathrm{~s}, T_{2}=1.5 \mathrm{~s}, D=1.85 \cdot 10^{-5} \mathrm{~cm}^{2} / \mathrm{s} ; \mathrm{CH}_{3} \mathrm{CN}$ : $T_{1}=2.0 \mathrm{~s}, T_{2}=2.3 \mathrm{~s}, D=0.55 \cdot 10^{-5} \mathrm{~cm}^{2} / \mathrm{s} ;\left(\mathrm{CH}_{3}\right)_{2} \mathrm{CO}: T_{1}=5.8 \mathrm{~s}, T_{2}=$ $5.2 \mathrm{~s}, D=1.28 \cdot 10^{-5} \mathrm{~cm}^{2} / \mathrm{s}$.

is valid, the slope of the log-log plot implied by Eq. [11] equals the coherence order $n$. Figure $3 b$ shows that the water data is described by Eq. [11].

The dependence of multiple coherence order detection on spin density is also reflected in a variety of simple solvent systems that have only a single detected resonance line (Fig. 3c). All data as well as simulations of these results demonstrate that the dominant effect on the observability of high-order coherences is the decline in the net equilibrium magnetization, $M_{0}$. 


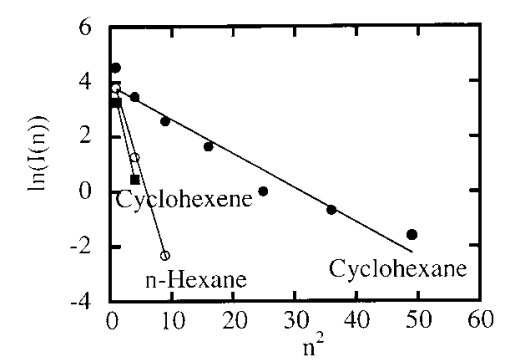

FIG. 4. The logarithm of the MQ signal intensity vs squared coherence order for hexane, cyclohexane, and cyclohexene, demonstrating the effects of chemical shifts and scalar couplings.

\section{B. Spectral Complexity Effects}

A related feature of the pulse sequence is the dependence of the echo intensity on the complexity of the spectrum. In the case where a single resonance line is observed, echoes of high order may be detected; however, when the spectrum has several lines (for example, due to resolved scalar couplings), only a few echoes are generally observed. This result may be qualitatively understood based on the partitioning of the total spin among different Larmor frequencies. In effect, the magnetization that enters Eq. [5] is the magnetization associated with each distinct line or Larmor frequency in the spectrum. Thus, for a doublet, the $n$ th-order intensity is reduced by $1 / 2^{n}$ and the attenuation proportionally larger for greater spectral partitioning of the magnetization. Furthermore, the distribution of Larmor frequencies makes dephasing much more rapid, and the sum of these effects is dramatic attenuation of high-order coherences. For example, Fig. 4 shows that seven echoes are readily observed for cyclohexane, while only two or three echoes are readily observed for $n$-hexane and cyclohexane, which have more complex spectra. We note that when scalar couplings are resolved, there is a competition between intramolecular $J$-couplings and intermolecular dipolar couplings. It was shown $(31,32)$ that the scalar contributions are in antiphase to the dipolar demagnetizing field effects, and the combination of the two types of couplings generates extra cross-peaks with the multiplet structures far different from conventional multiplets.

\section{Temperature Behavior of Multiple-Quantum Coherences}

Figure 5a shows data for water at two temperatures. At 283 $\mathrm{K}, 11$ echoes were detected, but at $323 \mathrm{~K}$ only 7 echoes appeared. The difference arises from changes in relaxation times, $T_{1}$ and $T_{2}$, effects of diffusion, and effects of temperature on the Boltzmann equilibrium magnetization. The persistence of high-order coherences depends on relatively long relaxation times. In water, both $T_{1}$ and $T_{2}$ increase with increasing temperature; thus, limitations from the relaxation times become less severe with increasing temperature and cannot explain the observations of Fig. 5a. The Boltzmann equilibrium magnetization changes by approximately $13 \%$ over the temperature range shown in Fig. 5a, which is significant because of the power law dependence of the $n$ th-order echo amplitude on $M_{0}$. The high-temperature data of Fig. 5a are similar to the $80 \% \mathrm{H}_{2} \mathrm{O}$ data of Fig. 3a; however, the equilibrium magnetization is somewhat higher. Thus, the effect of the equilibrium Boltzmann magnetization may account for most of the decline in observable high-order echoes with increasing temperature. The increasing effects of diffusion in the magnetic field gradients may account for the small additional decline. Thus, for rigid molecules, the increasing temperature will make high-order coherences more difficult to detect, partly because of increased effects of diffusion, but largely because of effects of the Curie law. Some discrepancy between numerical and experimental results can be accounted for by the magnetic field inhomogeneity effects as described above.

\section{Relaxation and Exchange Effects}

Cyclohexane demonstrates an opposite temperature behavior from water, i.e., more echoes are detected at high temperature. This behavior reflects the loss of phase coherence caused by time-dependence in the Larmor frequency. Cyclohexane is a classic fluxional molecule that was carefully characterized by Anet and Bourn (33). Figure 5b shows data for cyclohexane at several temperatures together with the results of numerical simulations. Although a single resonance line is generally
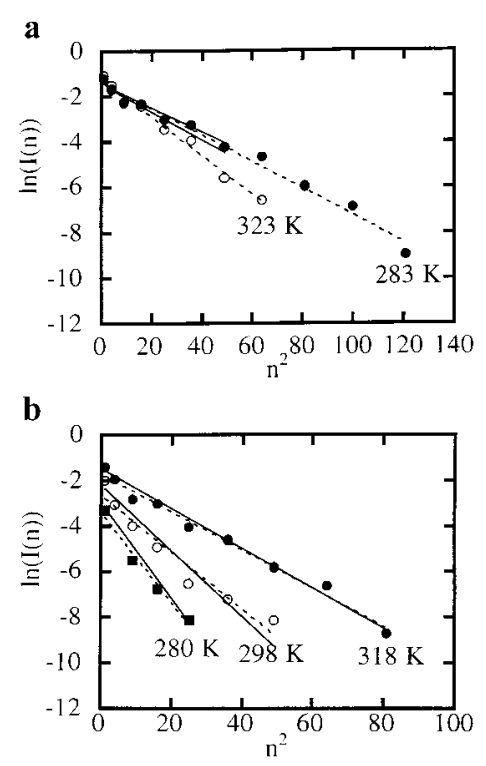

FIG. 5. (a) The logarithm of the MQ signal intensity vs squared coherence order for water at several temperatures. The solid lines represent the results of simulations while the dashed lines connect the experimental points. (b) The logarithm of the MQ coherence intensity vs squared coherence order for cyclohexane at several temperatures. The dashed lines connect experimental points; the solid lines refer to the simulations which were made with the following parameters: for $298 \mathrm{~K}: T_{1}=3.3 \mathrm{~s}, T_{2}=0.27 \mathrm{~s}, D=2.42 \cdot 10^{-5}$ $\mathrm{cm}^{2} / \mathrm{s}$; for $318 \mathrm{~K}: T_{1}=3.4 \mathrm{~s}, T_{2}=0.55 \mathrm{~s}, D=2.5 \cdot 10^{-5} \mathrm{~cm}^{2} / \mathrm{s}$; for 280 $\mathrm{K}: T_{1}=3.2 \mathrm{~s}, T_{2}=0.08 \mathrm{~s}, D=2.35 \cdot 10^{-5} \mathrm{~cm}^{2} / \mathrm{s}$. 


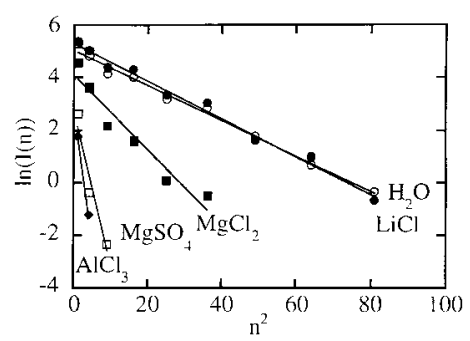

FIG. 6. The logarithm of the MQ coherence intensity vs squared coherence order for water in $1.0 \mathrm{M}$ solutions of $\mathrm{LiCl}, \mathrm{MgCl}_{2}, \mathrm{MgSO}_{4}$, and $\mathrm{AlCl}_{3}$.

observed, the chair-chair interconversion largely averages both axial-equatorial chemical shifts and the scalar coupling between these positions. Rates for the interconversion are on the order of $10^{5} \mathrm{~s}^{-1}$ at room temperature. As in other refocusing experiments, the time-dependence of the Larmor frequency, from whatever source, may interfere with the echo formation. The more rapid the conformational interconversion, the more completely the effective average Larmor frequency dominates the time evolution of the magnetization, and the longer the decay may persist. This effect is demonstrated in Fig. 5b. At $280 \mathrm{~K}$, the chair-chair interconversion rate is slower and only four echoes are detected. At $318 \mathrm{~K}$, the rate is more rapid and nine echoes are detected on the same sample. The experimental data in Fig. 5b fit quite well to the results of the simulations, which incorporate the effects of chemical exchange in the simulation at the level of changes in the transverse relaxation time that were measured experimentally. Short transverse relaxation time values and their strong temperature-dependence indicate that this parameter is mostly defined by the exchange effects.

Water is a particularly important solvent and provides a wide range of time-dependent interactions, particularly with simple electrolyte ions often present as buffers or supporting electrolyte species. Figure 6 shows several data sets for $1 \mathrm{M}$ solutions of several electrolytes. For simple alkali metal salts, the effects of echo attenuation are small; the results for lithium chloride are representative. The small effects for the alkali metal salts are expected because of the very rapid chemical exchange of water molecule protons between electrolyte ion coordination sites and the bulk aqueous environment. This exchange makes the effective Larmor frequency time-independent during the evolution times of the pulse sequence. However, the group II and group III metal ions are much less labile, which makes the chemical shift of the water resonance time-dependent as in the cyclohexane case. Thus, magnesium salts attenuate the water echoes significantly, and only three echoes are readily detected in the sulfate solution. The additional attenuation of the echo train by the sulfate ion relative to the chloride ion may be caused by the proton equilibrium with hydrogen sulfate ion, which provides an additional source of time-dependence in the proton resonance frequency. The effects for aluminum ion are dramatic. At a resonance frequency of $500 \mathrm{MHz}$, the water

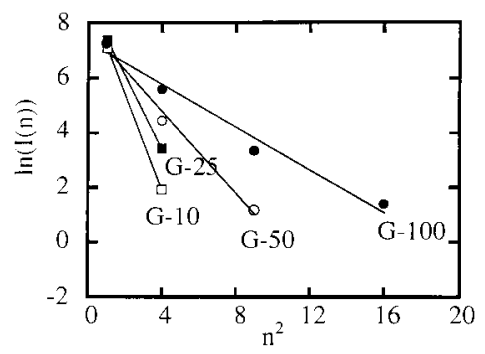

FIG. 7. The logarithm of the MQ signal intensity vs squared coherence order for water in samples of Sephadex G-10, G-25, G-50, and G-100.

resonance is significantly broadened by the exchange with the first aluminum coordination sphere, and only two echoes were detectable.

\section{E. Heterogeneous Systems}

Solutions of macromolecules and dynamically heterogeneous systems are important for many applications of these general approaches. Sephadex is a microporous carbohydrate used routinely for size-exclusion chromatography. Figure 7 shows data taken on Sephadex samples of different pore size. G-10 has the smallest pore size and G-100 the largest pore size. High-order coherences are attenuated in all samples relative to water. Part of the decrease in high-order echo observability may be caused by decreased water proton density; however, this cannot be the dominant effect. The initial echo intensity is nearly the same for all Sephadex samples, but the G-10 sample with the smallest pore size and highest surface area provides only two echoes while four echoes are observable in the G-100 sample. The Sephadex provides rotationally immobilized relaxation sites that affect both $T_{1}$ and $T_{2}$ (34). For Sephadex G-10 sample, $T_{2}$ was $0.018 \mathrm{~s}$. In this case, it is likely that exchange events involving very rapid transverse relaxation at bound water sites and chemical exchange with $\mathrm{OH}$ sites of the complex carbohydrate cause the significant drop in the water proton $T_{2}$. A consequence is that transverse magnetization does not last long enough to refocus higher order coherences.

In vivo applications involve protein solutes. Figure 8 summarizes data for bovine serum albumin samples at $7 \%$ by

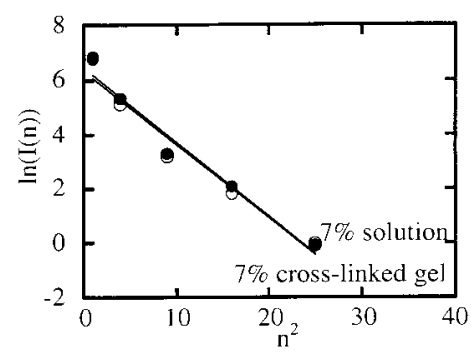

FIG. 8. The logarithm of the MQ coherence intensity vs squared coherence order for water in aqueous $7 \%$ bovine serum albumin and cross-linked $7 \%$ bovine serum albumin. 
weight. One is cross-linked by glutaraldehyde to provide a model for immobilized components of tissue. In both cases, the water echo train is attenuated relative to the protein-free case. There is a well-known magnetic coupling between the water protons and those of the protein which involves chemical exchange of labile protein protons as well as a few water molecules with specific sites on the protein (35). An important feature of Fig. 8 is that the results are the same for the cross-linked and the mobile protein cases. Changes in water proton $T_{1}$ are observable but relatively small at high fields when proteins are added because the effects of the protein largely disperse with the rotational correlation time of the protein in the range of several MHz. The present experiments are well above this relaxation dispersion. Effects on water proton $T_{2}$ are larger and are caused by the exchange of whole water molecules as well as protons with protein-binding sites. The integrated effect of these various exchange events, is to decrease $T_{2} . T_{2}$ will be somewhat shorter for the cross-linked gel, but apparently not sufficiently different to substantially attenuate the echo train. The protein solute may slow the translational diffusion of the water (34), which may decrease the effects of the field gradients at constant pulse separation. These effects are opposite to those observed. Thus, we conclude that the dominant effects in the protein system at high field are caused by changes in the water proton $T_{2}$.

\section{v. CONCLUSIONS}

This experimental study pushes the limits of the accepted theoretical model by exploring a wide range of magnetization densities, diffusion rates, and relaxation parameters. The experimental data are in good agreement with the results of the numerical simulations. These results demonstrate that highorder coherences may be detected even in CRAZED sequences that weight diffusion significantly. These data show for the first time that the log of the signal intensity is linearly proportional to the square of the coherence order for a wide range of conditions, similar to known results for intermolecular MQ coherences. In general, diffusion and residual inhomogeneous broadening cause this dependence, largely because of the relatively slow growth of signal from high-order MQ coherences. These data show that chemical exchange events that make the Larmor frequency time-dependent may be studied with high sensitivity. Finally, high-order coherences will be difficult to exploit in any system with a short transverse relaxation time such as heterogeneous systems; however, lower orders including zeroth-order coherences may be very fruitfully exploited.

\section{ACKNOWLEDGMENTS}

We acknowledge with pleasure stimulating discussions with J. Jeener. This work was supported by the NIH under Grants GM35253 (W²) and GM34541 (RGB).

\section{REFERENCES}

1. G. Bodenhausen, Prog. NMR Spectrosc. 14, 137 (1981).

2. D. P. Weitekamp, Adv. Magn. Reson. 11, 111 (1983).

3. M. Munowitz and A. Pines, Adv. Chem. Phys. 66, 1 (1985).

4. M. Munowitz and A. Pines, Science 233, 525 (1986).

5. R. R. Ernst, G. Bodenhausen, and A. Wokaun, "Principles of Nuclear Magnetic Resonance in One and Two Dimensions," Oxford, Clarendon, 1987.

6. S. Lacelle, Adv. Magn. Opt. Reson. 16, 173 (1991).

7. T. J. Norwood, Prog. NMR Spectrosc. 24, 295 (1992).

8. M. A. McCoy and W. S. Warren, J. Chem. Phys. 93, 858 (1990).

9. W. S. Warren and Q. He, J. Chem. Phys. 96, 1659 (1992).

10. Q. He, W. Richter, S. Vathyam, and W. S. Warren, J. Chem. Phys. 98, 6779 (1993).

11. W. S. Warren, W. Richter, A. H. Andreotti, and B. T. Farmer II, Science 262, 2005 (1993).

12. C. S. J ohnson, J r., Progr. NMR Spectrosc. 34, 203 (1999).

13. G. J . Bowden, T. Heseltine, and M. J. Prandolini, Chem. Phys. Lett. 233, 639 (1995).

14. W. S. Warren, S. Lee, W. Richter, and S. Vathyam, Chem. Phys. Lett. 247, 207 (1995).

15. M. H. Levitt, Concept. Magn. Reson. 8, 77 (1996).

16. S. Vathyam, S. Lee, and W. S. Warren, Science 272, 92 (1996).

17. W. S. Warren, S. Ahn, and M. Mescher, et al., Science 282, 247 (1998).

18. G. Deville, M. Bernier, and J. M. Delrieux, Phys. Rev. B 19, 5666 (1979).

19. D. Einzel, G. Eska, Y. Hirayoshi, T. Kopp, and P. Wolfe, Phys. Rev. Lett. 53, 2312 (1984).

20. R. Bowtell, R. M. Bowley, and P. Glover, J. Magn. Reson. 88, 643 (1990).

21. S. Lee, W. Richter, S. Vathyam, and W. S. Warren, J. Chem. Phys. 105, 874 (1996).

22. J. J eener, A. Vlassenbroek, and P. Broekaert, J . Chem. Phys. 103, 1309 (1995).

23. E. O. Stejskal, J. Chem. Phys. 43, 3597 (1965).

24. P. T. Callaghan, J. Magn. Reson. 88, 493 (1990).

25. S. Vega and A. Pines, J. Chem. Phys. 66, 5624 (1977).

26. G. Bodenhausen, R. L. Vold, and R. R. Vold, J. Magn. Reson. 37, 93 (1980).

27. D. Zax and A. Pines, J. Chem. Phys. 78, 6333 (1983).

28. N. Bloembergen and R. Pound, Phys. Rev. 95, 8 (1954).

29. T. Enss, S. Ahn, and W. S. Warren, Chem. Phys. Lett. 305, 101 (1999).

30. S. Garrett-Roe and W. S. Warren, J. Magn. Reson. 146, 1 (2000).

31. S. Ahn, S. Lee, and W. S. Warren, Mol. Phys. 95, 769 (1998).

32. S. Ahn and W. S. Warren, Chem. Phys. Lett. 29, 121 (1998).

33. F. A. L. Anet and A. J . R. Bourn, J. Am. Chem. Soc. 89, 760 (1967).

34. M. Whaley, A. J. Lawrence, J -P. Korb, and R. G. Bryant, Solid State NMR 7, 247-252 (1996).

35. R. G. Bryant, Ann. Rev. Biophys. Biomol. Struct. 25, 29-53 (1996). 